\title{
Novel Support Model for the Management of Occupational Stress Among Frontline Healthcare Workers During the COVID-19 Pandemic: a New Training Opportunity
}

\author{
Alana Warhit ${ }^{1}$ (D) Martin Ahern ${ }^{1} \cdot$ Michael L. Turman $^{1} \cdot$ Mariel Emrich $^{1} \cdot$ Jonathan Avery $^{1} \cdot$ Rosanne Raso $^{1} \cdot$ \\ JoAnn Difede ${ }^{1} \cdot$ Julie B. Penzner $^{2}$
}

Received: 29 January 2021 / Accepted: 7 April 2021 / Published online: 15 April 2021

(C) Academic Psychiatry 2021

To the Editor:

Occupational stress experienced by frontline healthcare workers during the COVID-19 pandemic is increasingly recognized [1]. These stressors include, but are not limited to, risk of personal injury and death due to exposure, isolation from social support systems, and physical exhaustion [2]. These stressors elevate healthcare workers' risk of experiencing psychiatric sequelae, ranging from adjustment disorders and acute stress reactions to depression, anxiety, and posttraumatic stress disorder [1].

To address the occupational stress of healthcare workers during the COVID-19 pandemic, New York Presbyterian Weill Cornell and Columbia University Medical Center created a cross-campus Psychiatric Liaison Program. Liaisons were mental health professionals, including physicians, psychologists, and social workers, with expertise in stress management, anxiety management, and coping with adversity. The main component of the program was group debriefings/check-ins conducted via tele-means. The objective of the check-ins was to provide a safe space for frontline workers to discuss their experiences during the pandemic. The liaisons facilitated discussions aimed at normalizing negative emotions and activating adaptive coping skills. They also identified specific healthcare workers who would benefit from additional services and referred them to mental health professionals.

The nursing community was identified as a high-risk population for occupational stress. Nursing responsibilities and stressors rapidly expanded, including being deployed to the Intensive Care Unit (ICU), having limited supply of personal

Alana Warhit

alw9092@nyp.org

1 Weill Cornell Medicine, New York, NY, USA

2 Duke University, Durham, NC, USA protective equipment, and having to facilitate video conferences between patients and their loved ones due to hospital visitor restriction policies. Nurses quickly expressed needing support "on the ground" in-person rather than through the video conference liaison model.

We implemented a model for psychiatry and psychology trainee-led, faculty-supervised, in-person individual and group debriefings. Faculty supervisors for the trainees also served as liaisons. The reason for the use of trainees, who were secondyear psychiatry residents and a fifth-year doctoral student in clinical psychology, as the nursing liaisons was primarily practical: many other staff involved in the liaison program were not hospital based. However, trainees were hospital based, accustomed to more irregular schedules, and had usual-care duties altered due to the surge of hospitalized COVID-19 patients. This presented a unique opportunity for trainees to provide support to nursing colleagues.

As a first step, specific units that would benefit from inperson nursing liaison check-ins were identified, and each unit was assigned one trainee. This enabled the trainee to familiarize him/herself with each unit's needs and to help establish a therapeutic alliance. Initially, each trainee met with his/her assigned unit at nursing change of shift to introduce him/ herself to both day and night shift nurses and the unit's Patient Care Director.

The Nursing Liaison program started on April 12, 2020, the week that Weill Cornell Medical Center experienced its peak number of COVID-19 inpatients, ICU-level patients, and mechanically ventilated patients. Initially, nursing liaisons visited higher acuity units two to three times per week and lower acuity units weekly. In the first 2 weeks of the program, very few nurses engaged with the liaisons about occupational stress. As nursing liaisons discussed the program's progress with unit Patient Care Directors, it became evident that some nurses were uncomfortable approaching liaisons during visits. In response, contact information was disseminated for each 
nursing liaison via email and flyers, encouraging nurses to reach out individually. During the third week, there was a noticeable increase in nursing engagement at unit check-ins, which coincided with a decrease in COVID-19 patient admissions. We suspect that nurses had more time to engage with the trainees, which also resulted in rapport-building and alliance development.

Unforeseen needs arose as the program progressed. There was increased demand to provide support to night-shift nurses and to provide unit-specific support surrounding one unit's loss of a nursing colleague due to COVID-19. In response, the nursing liaisons held early morning check-ins for nightshift nurses, and an additional group was held to provide grief support. As the volume of hospitalized COVID-19 patients decreased by mid-May, and due to scheduling constraints of the trainees, frequency of unit visits was reduced, but the nursing liaisons remained available for individual outreach.

Over the course of 3 months, the nursing liaisons and faculty supervisors conducted 522 check-ins with 37 different units in the hospital. They spoke to approximately 1041 individuals either by tele-means or in-person over the course of this program.

We found that the in-person check-in model successfully engaged nurses. Feedback from nursing was that the regularity of the check-ins made them feel supported and provided them a safe space to voice their concerns. This created a framework within which nurses could access support and process their emotions. We believe this can be attributed to the development of a significant therapeutic alliance, which has been shown to be a major variable in the outcome of psychological treatment $[3,4]$. Themes that arose included fear of spreading and/or contracting the virus, feelings of guilt, sadness, loneliness, anger, and frustration.

Interestingly, although trainees were selected as the nursing liaisons for practical reasons, unintended benefits arose. Specifically, the trainees expressed that they learned to deliver support in a time-limited fashion, establish rapport and normalize reactions to occupational stress, offer both short-term and long-term coping skills, and activate additional support systems. At times, the trainees felt it was challenging to accomplish these goals in the time-limited setting, and, therefore, supervision, discussion, and refinement of technique among trainees were key.

There were certain limitations to our program. Many nurses were unavailable during liaison visits. Perhaps, complementing in-person check-ins with set video conference check-ins would allow for more nurse participation. Additionally, although many nursing units continued to want in-person check-ins with their assigned liaison, this was not possible as trainees, who require a diversity of experiences and rotations, had to transition to other roles. Another limitation was that many nurses may have developed delayed psychiatric sequelae and, given the limited time frame of our intervention program, were not able to be identified by the liaisons.

We believe that our in-person nursing liaison program could be adapted to other healthcare settings and is a unique educational opportunity for psychiatry and psychology trainees. Future work may focus on the applicability and utility of this model to other disaster situations.

Acknowledgment The authors have informed the journal that they agree that both Alana Warhit, MD, and Martin Ahern, MD, completed the intellectual and other work typical of the first author.

\section{Declarations}

Disclosure On behalf of all authors the corresponding author states that there is no conflict of interest.

\section{References}

1. Lai J, Ma S, Wang Y, Cai Z, Hu J, Wei N, et al. Factors associated with mental health outcomes among health care workers exposed to coronavirus disease 2019. JAMA Netw Open. 2020;3(3):e203976. Available from: https://jamanetwork.com/journals/ jamanetworkopen/fullarticle/2763229. https://doi.org/10.1001/ jamanetworkopen.2020.3976.

2. Krystal JH. Responding to the hidden pandemic for healthcare workers: stress. Nat Med. 2020;26:639. https://doi.org/10.1038/ s41591-020-0878-4.

3. Catty J. 'The vehicle of success': theoretical and empirical perspectives on the therapeutic alliance in psychotherapy and psychiatry. Psychol Psychother. 2010;77(2):255-72. https://doi.org/10.1348/ 147608304323112528.

4. Elvins R, Green J. The conceptualization and measurement of therapeutic alliance: an empirical review. Clin Psychol Rev. 2008;28(7): 1167-87. https://doi.org/10.1016/j.cpr.2008.04.002.

Publisher's Note Springer Nature remains neutral with regard to jurisdictional claims in published maps and institutional affiliations. 